\title{
Dexmedetomidine attenuates lipopolysaccharide-induced acute lung injury by inhibiting oxidative stress, mitochondrial dysfunction and apoptosis in rats
}

\author{
CHUNLAI FU ${ }^{1,2^{*}}$, XINGUI DAI $^{2^{*}}$, YOU YANG $^{3 *}$, MENGXIANG LIN $^{4}$, YEPING CAI $^{2}$ and SHAOXI CAI \\ ${ }^{1}$ Chronic Airways Diseases Laboratory, Department of Respiratory and Critical Care Medicine, Nanfang Hospital, \\ Southern Medical University, Guangzhou, Guangdong 510515; ${ }^{2}$ Department of Critical Care Medicine; \\ ${ }^{3}$ Medical Imaging Center, The First People's Hospital of Chenzhou, Institute of Translation Medicine, \\ Chenzhou, Hunan 423000; ${ }^{4}$ Department of Critical Care Medicine, The Second Affiliated Hospital \\ of Wenzhou Medical University, Wenzhou, Zhejiang 325000, P.R. China
}

Received October 25, 2015; Accepted November 3, 2016

DOI: $10.3892 / \mathrm{mmr} .2016 .6012$

\begin{abstract}
Previous studies have identified that dexmedetomidine (DEX) treatment can ameliorate the acute lung injury (ALI) induced by lipopolysaccharide and ischemia-reperfusion. However, the molecular mechanisms by which DEX ameliorates lung injury remain unclear. The present study investigated whether DEX, which has been reported to exert effects on oxidative stress, mitochondrial permeability transition pores and apoptosis in other disease types, can exert protective effects in lipopolysaccharide (LPS)-induced ALI by inhibiting oxidative stress, mitochondrial dysfunction and mitochondrial-dependent apoptosis. It was revealed that LPS-challenged rats exhibited significant lung injury, characterized by the deterioration of histopathology, vascular hyperpermeability, wet-to-dry weight ratio and oxygenation index $\left(\mathrm{PaO}_{2} / \mathrm{FIO}_{2}\right)$, which was attenuated by DEX treatment. DEX treatment inhibited LPS-induced mitochondrial dysfunction, as evidenced by alleviating the cellular ATP and mitochondrial membrane potential in vitro. In addition, DEX treatment markedly prevented the LPS-induced mitochondrial-dependent apoptotic pathway in vitro (increases of cell apoptotic rate, cytosolic cytochrome $c$, and caspase 3 activity) and in vivo (increases of Iterminal deoxynucleotidyl transferase dUTP nick-end labeling positive cells, cleaved caspase 3, Bax upregulation and Bcl-2 downregulation). Furthermore,
\end{abstract}

Correspondence to: Dr Shaoxi Cai, Chronic Airways Diseases Laboratory, Department of Respiratory and Critical Care Medicine, Nanfang Hospital, Southern Medical University, 1838 Guangzhou Avenue North, Guangzhou, Guangdong 510515, P.R. China

E-mail: caishaox@fimmu.com

${ }^{*}$ Contributed equally

Key words: dexmedetomidine, lipopolysaccharide, sepsis, lung injury, oxidative stress, mitochondria, apoptosis
DEX treatment markedly attenuated LPS-induced oxidative stress, as evidenced by downregulation of cellular reactive oxygen species in vitro and lipid peroxides in serum. Collectively, the present results demonstrated that DEX ameliorates LPS-induced ALI by reducing oxidative stress, mitochondrial dysfunction and mitochondrial-dependent apoptosis.

\section{Introduction}

Acute lung injury (ALI) and acute respiratory distress syndrome are well-defined and readily recognized clinical disorders caused by numerous clinical insults to the lung or due to predispositions to lung injury (1-3). ALI is a frequent complication following sepsis in critically ill patients and lipopolysaccharide (LPS) is thought to be the most important pathogen that leads to the development of ALI in sepsis $(4,5)$.

Mitochondrial damage is associated with numerous human diseases, including ALI (6-9). Mitochondria are involved and serve a central role in the integration and circulation of death signals initiating inside the cells, including oxidative stress and DNA damage, and in regulating cell death/apoptosis pathways (10-12). Mitochondrial regulation of apoptosis is predominantly mediated via the release of cytochrome $c$, apoptosis-inducing factor (AIF), and second mitochondria-derived activator of caspases (Smac) and ultimately caspase activation (13-15).

Dexmedetomidine (DEX), a highly selective and potent $\alpha 2$-adrenoreceptor agonist, provides excellent sedation and analgesia with minimal cardiovascular effects. Previous studies have shown that DEX attenuates LPS, ischemia-reperfusion and ventilator-induced lung injury in animal models; however, the mechanism remains unclear (16-19). Additionally, the anti-inflammatory and antiapoptotic effects of DEX have been demonstrated in previous studies (20-23). DEX has also been reported to have an effect on mitochondrial permeability transition pore (mPTP) in neutrophil and isolated rat hearts following ischemia/reperfusion injury (24-26). The present study hypothesized that DEX may provide protective effect 
against LPS-induced ALI by alleviating oxidative stress, mitochondrial damage and mitochondria-dependent apoptosis.

\section{Materials and methods}

Animals. All experimental protocols were approved by the Animal Care and Use Committee of the Southern Medical University (Guangzhou, China). The care of animals was in accordance with the National Institute of Health guidelines and with those of the Chinese National guidelines. A total of 24 Male Sprague-Dawley rats (weight, 180-220 g) were purchased from the Experimental Animal Center at South Medical University (Guangzhou, China) and were allowed to acclimate for 1 week prior to experiments. Animals were housed in individual cages in a temperature-controlled $\left(25 \pm 2^{\circ} \mathrm{C}\right)$ room, under a $12 \mathrm{~h} \mathrm{light/dark} \mathrm{cycle,} \mathrm{with} \mathrm{ad} \mathrm{libitum}$ access to food and water.

Animal model. As previously described (27), ALI was induced by intratracheal administration of LPS. Briefly, the animals were intramuscularly anesthetized with an injection of sodium pentobarbital $(30 \mathrm{mg} / \mathrm{kg})$. The rats were placed in a supine position on a warming device and the trachea was surgically exposed by a cervical middle line incision in the skin. The rats were subsequently challenged intratracheally with either $0.5 \mathrm{ml}$ sterile normal saline (NS) alone or $0.5 \mathrm{ml}$ NS with LPS (5 mg/kg body weight; Escherichia coli 0111:B4; Sigma-Aldrich; Merck Millipore, Darmstadt, Germany) stabbing the trachea with a microsyringe. Prior to ALI induction, the rats were pretreated with DEX (10 or $50 \mu \mathrm{g} / \mathrm{kg}$ ) for $30 \mathrm{~min}$. NS was used as the vehicle control.

Cell culture and stimulation. Rat type I alveolar epithelial cells (AECs) were obtained from ScienCell (San Diego, CA, USA) and grown at $37^{\circ} \mathrm{C}$ in $5 \% \mathrm{CO}_{2}$ in Dulbecco's modified Eagle medium (DMEM, Sigma-Aldrich; Merck Millipore), containing low glucose, penicillin (100 U/ml, Sigma-Aldrich; Merck Millipore), streptomycin (100 units, Sigma-Aldrich; Merck Millipore) and 10\% bovine serum (Sigma-Aldrich; Merck Millipore). The AECs were respectively pretreated with 10 and $50 \mu \mathrm{g} / \mathrm{l} \mathrm{DEX}$ for $30 \mathrm{~min}$, followed by stimulation of $5 \mu \mathrm{g} / \mathrm{ml}$ LPS for $12 \mathrm{~h}$. DMEM was used as a vehicle control.

Experimental design. In vivo, rats were intramuscularly anesthetized with an injection of sodium pentobarbital $(30 \mathrm{mg} / \mathrm{kg})$, and were then sacrificed by decollation at $24 \mathrm{~h}$ post-LPS treatment. The lung injury oxygenation index $\left(\mathrm{PaO}_{2} / \mathrm{FIO}_{2}\right)$, histopathological changes, lung microvascular permeability and wet-to-dry (W/D) weight ratio were measured. To investigate the underlying mechanisms of DEX treatment in LPS-induced ALI, terminal deoxynucleotidyl transferase dUTP nick end labeling (TUNEL) staining, serum lipid peroxidation, caspase 3 activity, and the expression levels of Bax, Bcl-2 and cleaved caspase 3 were investigated.

In vitro, following $12 \mathrm{~h}$ stimulation, oxidative stress, as determined by reactive oxygen species (ROS) production, mitochondrial function, as determined by mitochondrial membrane potential (MMP) and cellular ATP, and mitochondria-dependent apoptosis, as determined by cell apoptotic rate, cytochrome $c$ release and caspase-3 activity were detected.

Histological examination. The lungs were harvested $24 \mathrm{~h}$ following LPS injection. The right middle lobes of the lungs were fixed with $10 \%$ formalin, embedded in paraffin and sectioned (4 $\mu \mathrm{m}$ thickness). Following deparaffinization and rehydration, the tissue sections were stained with hematoxylin and eosin. The pathological sections were observed and assessed in a blinded manner.

Lung microvascular permeability assay. The permeability assay was performed, as previously described (4). Briefly, Evans blue (EB; $20 \mathrm{mg} / \mathrm{kg}$; Sigma-Aldrich) was injected intravenously through the femoral vein. After $30 \mathrm{~min}$, the animals were intramuscularly anesthetized with an injection of sodium pentobarbital $(30 \mathrm{mg} / \mathrm{kg})$, and were then sacrificed by decollation, and a midline thoracotomy was performed. The superior and inferior vena cava were subsequently ligated, the aorta was transected, and $20 \mathrm{ml}$ normal saline solution was injected into the right ventricle at a pressure of $20 \mathrm{~cm} \mathrm{H}_{2} \mathrm{O}$ to wash out the pulmonary intravascular content. A sample of lung tissue was weighed, homogenized and immersed in $\mathrm{N}, \mathrm{N}$-dimethylformamide (Sigma-Aldrich). The homogenate was incubated at room temperature for $48 \mathrm{~h}$. Eluted EB was measured at $620 \mathrm{~nm}$ using an automatic microplate reader (SpectraMax M5; Molecular Devices, Sunnyvale, CA, USA), and the quantity was expressed as $\mu \mathrm{g} / 100 \mathrm{mg}$ dry tissue.

Measurement of lung W/D weight ratio. The harvested wet lung was weighed and subsequently placed in an oven for $48 \mathrm{~h}$ at $80^{\circ} \mathrm{C}$, followed by weighing when it was dried. The lung $\mathrm{W} / \mathrm{D}$ weight ratio was calculated.

Oxygenation index $\left(\mathrm{P}_{a} \mathrm{O}_{2} / \mathrm{F}_{i} \mathrm{O}_{2}\right)$ analysis. At $24 \mathrm{~h}$ following ALI (or sham), animals were anesthetized and administered endotracheal intubation with a 20 -gauge catheter. The animals were mechanically ventilated with pure oxygen at $7 \mathrm{ml} / \mathrm{kg}$ (120 breaths/min). Following 20 min ventilation, the arterial blood was obtained from the carotid artery and measured using a commercial blood gas analyzer (ABL8000; Radiometer Copenhagen, Copenhagen, Denmark).

Measurement of mitochondrial membrane potential $(M M P)$ in vitro. The MMP was determined using the potential-sensitive fluorescent dye, JC-1. This dual-emission probe changes color from red-orange to green as the mitochondrial membrane depolarizes. The JC-1 $(5 \mu \mathrm{mol} / \mathrm{l})$ was loaded onto AECs for $15 \mathrm{~min}$ at $37^{\circ} \mathrm{C}$. The results were visualized using a confocal microscope (x650; LSM 780 NLO; Carl Zeiss, Jena, Germany). Alternatively, the fluorescence intensity was monitored using flow cytometry (BD Immunocytometry Systems, Franklin Lakes, NJ, USA) at an excitation/emission of $525 / 590 \mathrm{~nm}$.

Measurement of cellular ATP in vitro. Intracellular ATP was determined using a luciferase-based assay (CellTiter-Glo, Madison, WI, USA), according to the manufacturer's protocol. Following the addition of $100 \mu \mathrm{l}$ CellTiter-Glo reagent to 
$100 \mu 1$ cell suspension containing 10,000 cells in each well of a standard opaque-walled 96-well plate, the plates were allowed to incubate at room temperature for $10 \mathrm{~min}$, and the luminescence was recorded in an automatic microplate reader (SpectraMax M5; Molecular Devices).

TUNEL staining. Lung histopathological slides were dewaxed and incubated with proteinase $\mathrm{K}$. The slides were stained using a TUNEL kit (Biovision, Mountain View, CA, USA), according to the manufacturer's instructions. Subsequently, the cells were counterstained with Hoechst 33258 (Sigma-Aldrich) and examined under a fluorescence microscope (ECLIPSE FN1, Nikon).

Measurement of cell apoptosis in vitro. Cell apoptosis was detected using an annexin V-FITC apoptosis detection kit (BD Biosciences, Franklin Lakes, NJ, USA). Following induction with $\mathrm{H}_{2} \mathrm{O}_{2}$, the cells $\left(\sim 1 \times 10^{5}\right.$ cells $\left./ \mathrm{ml}\right)$ were washed twice with PBS and suspended in $1 \mathrm{X}$ binding buffer. A total of $5 \mu \mathrm{l}$ annexin V-FITC and $10 \mu \mathrm{l}$ propidium iodide (PI; $50 \mu \mathrm{g} / \mathrm{ml}$; Sigma-Aldrich) was added to the cell suspension. Following incubation at room temperature for $20 \mathrm{~min}$ in the dark, the fluorescence of the cells was determined immediately using a flow cytometer (BD Immunocytometry Systems).

Measurement of cytosolic cytochrome $c$ in vitro. Cytosolic cytochrome $c$ content was estimated using a cytochrome $c$ ELISA kit (Biovision), as described previously (28). The cell homogenates were centrifuged $(10,000 \times \mathrm{g}$ for $60 \mathrm{~min}$ at $4^{\circ} \mathrm{C}$ ) and the supernatant (cytosolic fraction) was collected and subjected to protein estimation using the bicinchoninic acid method (BCA). The samples were treated with a conjugate reagent, transferred to a cytochrome $c$ antibody-coated microwell plate and incubated at room temperature for $60 \mathrm{~min}$. The wells were washed and treated with a substrate and incubated for $30 \mathrm{~min}$ at room temperature, followed by the addition of a stop solution. The optical density was measured at $450 \mathrm{~nm}$ using an automatic microplate reader (SpectraMax M5). A serial dilution of cytochrome $c$ calibrator was subjected to the assay, along with the samples. The values were plotted and the concentration of cytochrome $c$ was calibrated from the standard curve.

Measurement of caspase 3 activity in vitro. Caspase 3 activity was determined using a caspase 3 activity assay kit, according to the manufacturer's instructions (Biovision). The cells were lysed in caspase 3 sample lysis buffer. The homogenates were centrifuged at 10,000 $\mathrm{x} g$ and the supernatant was collected for protein estimation using BCA and for the caspase 3 assay. The cell lysates were exposed to the DEVD substrate conjugate provided in the kit. The sample was measured in an automatic microplate reader (SpectraMax M5) at excitation of $400 \mathrm{~nm}$ and emission of $505 \mathrm{~nm}$.

Western blot analysis for Bax, Bcl-2 and cleaved caspase 3 in vivo. The lung tissues were homogenized and analyzed by western blotting. Protein concentrations were determined using the BCA method. An equal quantity of protein was loaded onto $10 \%$ sodium dodecyl sulphate-polyacrylamide gels for electrophoresis. Following electrophoresis, the proteins were electroblotted onto polyvinylidene difluoride membranes. Membranes were blocked with blocking solution (5\% skimmed milk diluted with PBS) at room temperature for $2 \mathrm{~h}$, followed by incubation with primary antibodies against $\beta$-actin (1:5000 dilution; cat. no. ab8227; Abcam, Cambridge, UK), Bax (1:1,000 dilution; cat. no. ab32503; Abcam), Bcl-2 (1:1,000 dilution; cat. no. ab59348; Abcam) and cleaved caspase 3 (1:1,000 dilution; cat. no. ab13847; Abcam) overnight at $4^{\circ} \mathrm{C}$. The membranes were subsequently incubated with horseradish peroxidase-conjugated secondary antibody (1:5,000, cat. no. ab6721, Abcam), and the protein expression was detected using an enhanced chemiluminescence reagent [cat. no. abs920B-500, Absin Biotechnology Co., Ltd., Shanghai, China].

Measurement of ROS levels in vitro. Intracellular ROS levels were assessed using a DCFH-DA probe (Sigma-Aldrich). The cells were treated with DCFH-DA $(10 \mu \mathrm{M})$ after LPS stimulation for $20 \mathrm{~min}$ at $37^{\circ} \mathrm{C}$. Following incubation, the cells were washed and analyzed using an automatic microplate reader (SpectraMax M5). The relative intensity of DCF fluorescence was determined at a wavelength of $535 \mathrm{~nm}$, as compared with the sham group cells.

Measurement of lipid peroxides in vivo. The serum was obtained as described above. Lipid peroxides (LPOs) were measured using a commercially available kit (Cayman Chemical Co., Ann Arbor, MI, USA), according to the manufacturer's protocol. The LPO content was measured at $500 \mathrm{~nm}$ using an automatic microplate reader (SpectraMax M5).

Statistical analysis. All variables are presented as the mean \pm standard deviation. Differences between the groups were determined using one-way analysis of variance with the least significant difference multiple-comparison test and Student's $t$-test when appropriate. $\mathrm{P}<0.05$ was considered to indicate a statistically significant difference.

\section{Results}

DEX attenuates LPS-induced ALI in rats. The present study examined the effects of DEX on LPS-induced ALI in rats. Lung histological sections of LPS-challenged rats exhibited accumulation of a large number of neutrophils in the intra and interalveolar space, a thickened alveolar wall, less alveolar space and interstitial congestion, and these alterations were markedly attenuated by pretreatment with DEX $(50 \mu \mathrm{g} / \mathrm{kg})$ (Fig. 1A). Additionally, the lung microvascular permeability, reflected by EB content in lung tissue and W/D weight ratio of the lung were increased in LPS-challenged rats compared with the control animals. DEX pretreatment reduced the EB content in lung and the W/D weight ratio of lung compared with the LPS group $(\mathrm{P}<0.05$ compared with the LPS group for $50 \mu \mathrm{g} / \mathrm{kg}$ DEX group; P $>0.05$ compared with the LPS group for $10 \mu \mathrm{g} / \mathrm{kg}$ DEX group; Fig. 1B-D). In addition, the $\mathrm{P}_{\mathrm{a}} \mathrm{O}_{2} / \mathrm{F}_{\mathrm{i}} \mathrm{O}_{2}$ was significantly decreased in LPS-challenged rats, which was improved by $\mathrm{DEX}$ treatment $(\mathrm{P}<0.05$ compared with the LPS group for $50 \mu \mathrm{g} / \mathrm{kg}$ DEX group; P>0.05 compared with the LPS group for $10 \mu \mathrm{g} / \mathrm{kg}$ DEX group; Fig. 1D). These results indicated that DEX pretreatment attenuated LPS-induced ALI in rats. 
A

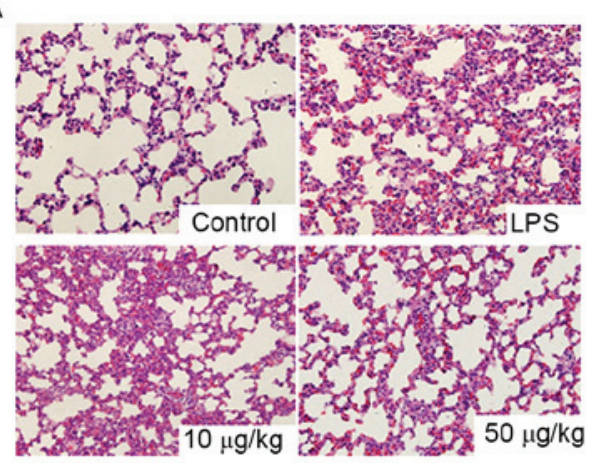

C

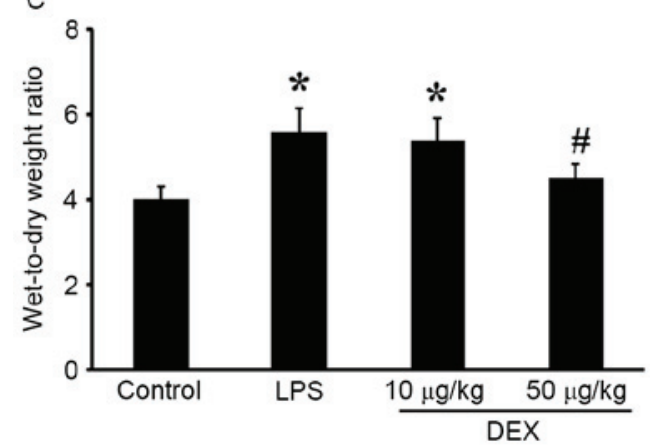

B

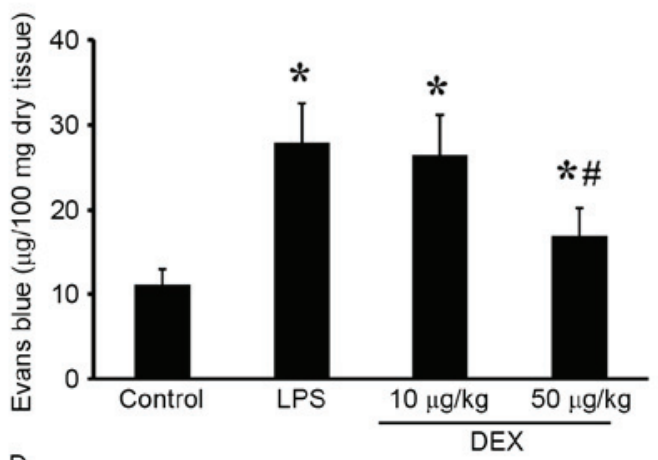

D

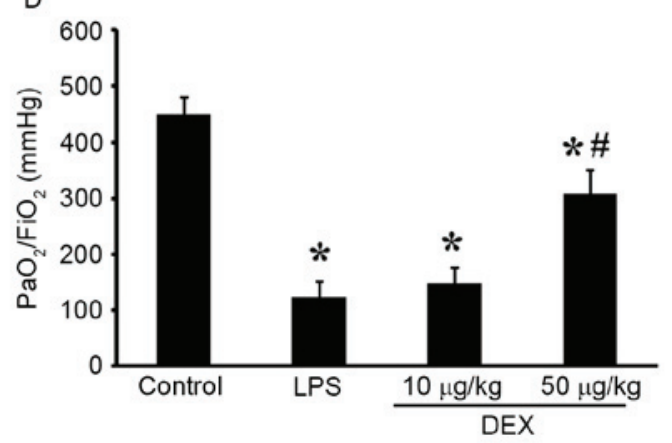

Figure 1. DEX improves LPS-induced lung injury in rat. (A) Comparison of histopathological changes using hematoxylin and eosin staining in control samples, LPS-treated samples and various concentrations of DEX. The lung in the LPS group exhibited a thickened alveolar wall, edema, less alveolar space and clear inflammatory cell infiltration. DEX (50 $\mu \mathrm{g} / \mathrm{kg})$ significantly prevented the histopathological changes caused by LPS. (B) DEX (50 $\mu \mathrm{g} / \mathrm{kg})$ significantly prevented LPS-induced vascular hyperpermeability, as demonstrated using Evans blue content in the lung. (C) DEX (50 $\mu \mathrm{g} / \mathrm{kg}$ ) significantly prevented LPS-induced lung edema, as determined by the Wet-to-dry lung weight ratio. (D) DEX (50 $\mu \mathrm{g} / \mathrm{kg}$ ) significantly prevented LPS-induced lung dysfunction, as determined by the oxygenation index $\left(\mathrm{PaO}_{2} / \mathrm{FIO}_{2}\right)$. The data are presented as the mean \pm standard deviation $(\mathrm{n}=6 / \mathrm{group}$; $\mathrm{P}<0.05$ compared with the control group; ${ }^{\text {P }}<0.05$ compared with the LPS group). DEX, dexmedetomidine; LPS, lipopolysaccharide.

DEX prevents LPS-induced mitochondrial dysfunction. To establish whether DEX attenuated LPS-induced mitochondrial dysfunction, the level of MMP and cellular ATP levels were evaluated. JC-1, the potential-sensitive fluorescent dye, forms aggregates in normally polarized mitochondria and monomers in damaged and depolarized mitochondria. The color of this dual-emission probe changed from red-orange to green as the mitochondrial membrane turned depolarized. As shown in Fig. 2A and B, the control cells were clearly red. However, LPS exposure rapidly caused MMP dissipation, as shown by the increase in green fluorescence and the concomitant disappearance of red fluorescence. Pretreatment with DEX significantly attenuated the changes in MMP, as indicated by the repression of green fluorescence and restoration of red fluorescence $(\mathrm{P}<0.05$ compared with the LPS group for $50 \mu \mathrm{g} / 1 \mathrm{DEX}$ group; $\mathrm{P}>0.05$ compared with the LPS group for $10 \mu \mathrm{g} / 1$ DEX group). In addition, the intracellular ATP level was decreased by LPS stimulation compared with the control group, indicating mitochondrial dysfunction in the AECs. Following pretreatment with the DEX, the ATP levels were increased compared with the LPS group $(\mathrm{P}<0.05$ compared with the LPS group for $50 \mu \mathrm{g} / 1 \mathrm{DEX}$ group; $\mathrm{P}>0.05$ compared with the LPS group for $10 \mu \mathrm{g} / 1 \mathrm{DEX}$ group; Fig. 2C).

DEX inhibits the LPS-induced mitochondrial-dependent apoptosis. To determine the protective effects of DEX against
LPS-induced apoptosis, TUNEL and annexin-V/PI double staining assays were performed. In vivo, LPS-challenged animals exhibited a significant increase in red marked apoptotic cells, which was reduced by DEX $(50 \mu \mathrm{g} / \mathrm{kg})$ pretreatment (Fig. 3A and B). In vitro, the rates of cell apoptosis were markedly increased following LPS exposure, compared with the control group, and were subsequently reduced by DEX pretreatment $(\mathrm{P}<0.05$ compared with the LPS group for $50 \mu \mathrm{g} / 1$ DEX group; P>0.05 compared with the LPS group for $10 \mu \mathrm{g} / 1$ DEX group; Fig. 3C).

Mitochondrial regulation of apoptosis is mediated through the release of cytochrome $c$, AIF and Smac, and ultimately caspase activation. In present study, cytochrome $c$ release, caspase 3 activity and cleaved caspase 3 expression were detected. As shown in Fig. 4, increased cytosolic cytochrome $c$ and caspase 3 activities were observed in vitro, which was reduced by DEX pretreatment $(\mathrm{P}<0.05$ compared with the LPS group for $50 \mu \mathrm{g} / 1$ DEX group; $\mathrm{P}>0.05$ compared with the LPS group for $10 \mu \mathrm{g} / 1$ DEX group). In the lungs, pretreatment with DEX $(50 \mu \mathrm{g} / \mathrm{kg})$ inhibited LPS-induced cleaved caspase 3 upregulation (Fig. 5A and $\mathrm{B} ; \mathrm{P}<0.05$ compared with the LPS group for $50 \mu \mathrm{g} / \mathrm{kg}$ DEX group; $\mathrm{P}>0.05$ compared with the LPS group for $10 \mu \mathrm{g} / \mathrm{kg}$ DEX group).

Additionally, the present study investigated the changes in expression levels of the Bcl-2 family of proteins (Bax and $\mathrm{Bcl}-2)$ in lung tissue. LPS inhalation resulted in downregulation of the Bcl-2 protein, and DEX prevented this decrease $(\mathrm{P}<0.05$ 

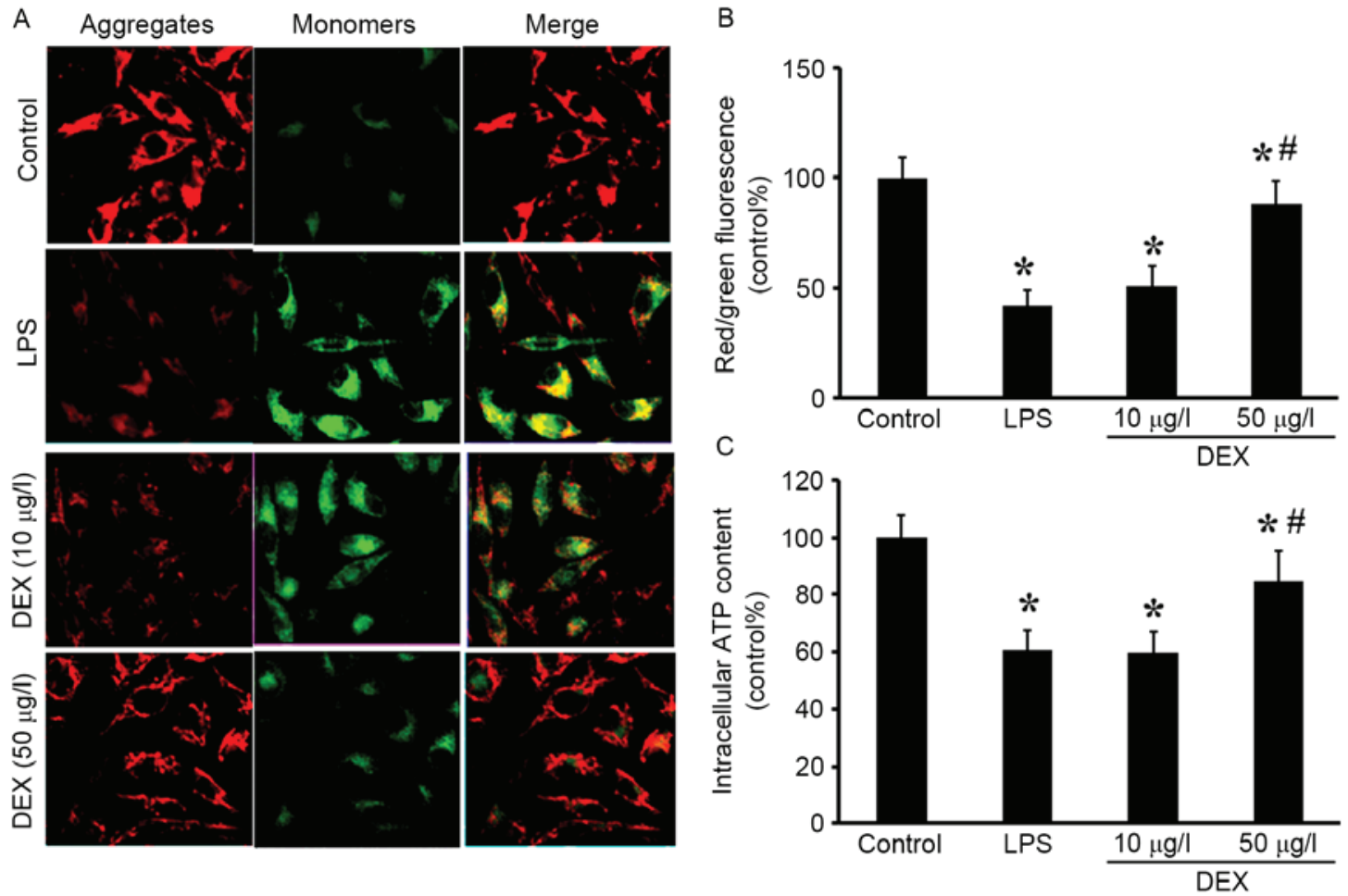

Figure 2. DEX improves LPS-induced mitochondrial dysfunction in vitro. (A) Intracellular red and green fluorescence of JC-1 was determined using a confocal microscope. The aggregates and monomers were assessed in the control, LPS and DEX (10 and $50 \mu \mathrm{g} / \mathrm{l})$ groups. (B) The intracellular red and green fluorescence of JC-1 was measured by flow cytometry in the various groups. (C) The ATP level of the alveolar epithelial cells was determined using a luciferase-based assay. The data are presented as the mean \pm standard deviation ( $\mathrm{n}=6 /$ group; ${ }^{*} \mathrm{P}<0.05$ compared with the control group; ${ }^{*} \mathrm{P}<0.05$ compared with the LPS group). DEX, dexmedetomidine; LPS, lipopolysaccharide.
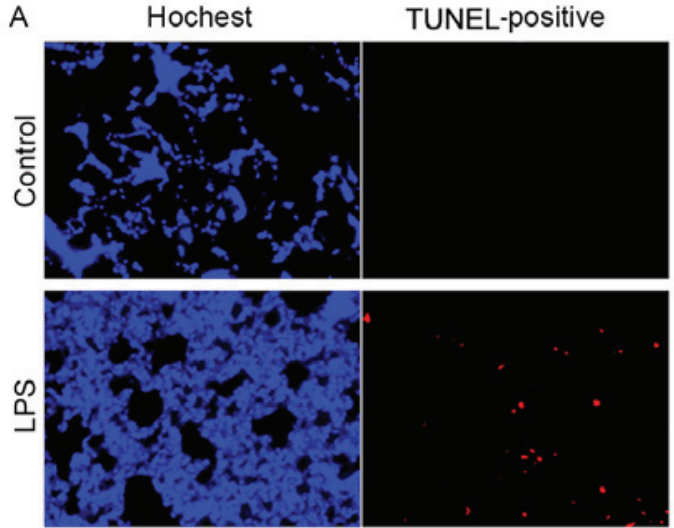

B
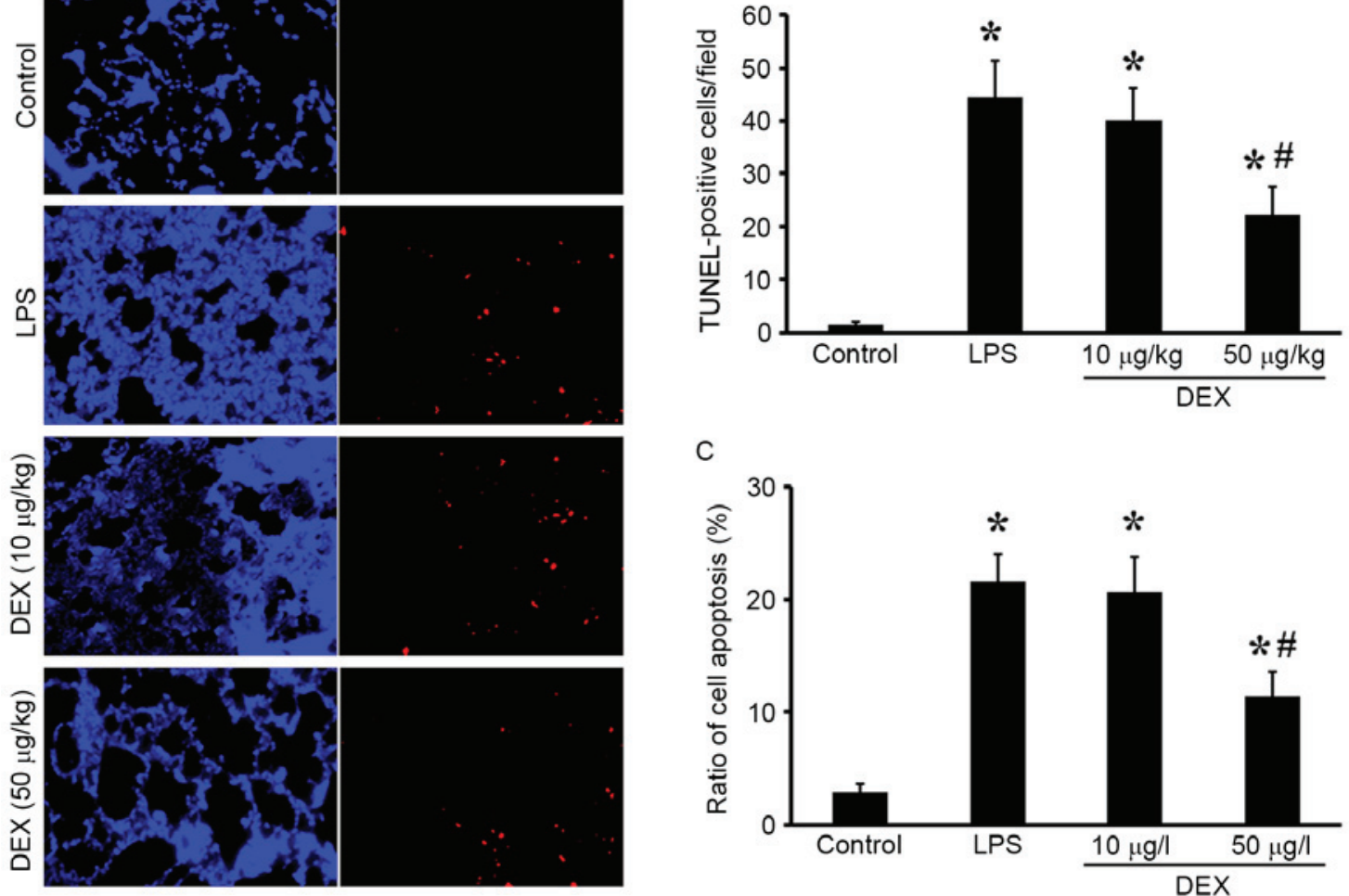

Figure 3. DEX improves LPS-induced apoptosis in vivo and in vitro. (A) Images of TUNEL staining in the control, LPS and DEX (10 and $50 \mu \mathrm{g} / \mathrm{kg}$ ) groups were captured using fluorescent microscopy (magnification, x200). (B) Graphical representation of TUNEL-positive cells, averaged over 10 microscopic fields per animal. (C) The ratio of alveolar epithelial cell apoptosis, as assessed using an annexin V/propidium iodide double stain assay, in the control, LPS and DEX (10 and $50 \mu \mathrm{g} / \mathrm{l})$ groups. The data are presented as the mean \pm standard deviation ( $\mathrm{n}=6 /$ group; ${ }^{*} \mathrm{P}<0.05$ compared with the control group; ${ }^{*} \mathrm{P}<0.05$ compared with the LPS group). DEX, dexmedetomidine; LPS, lipopolysaccharide; TUNEL, terminal deoxynucleotidyl transferase dUTP nick-end labeling. 
A
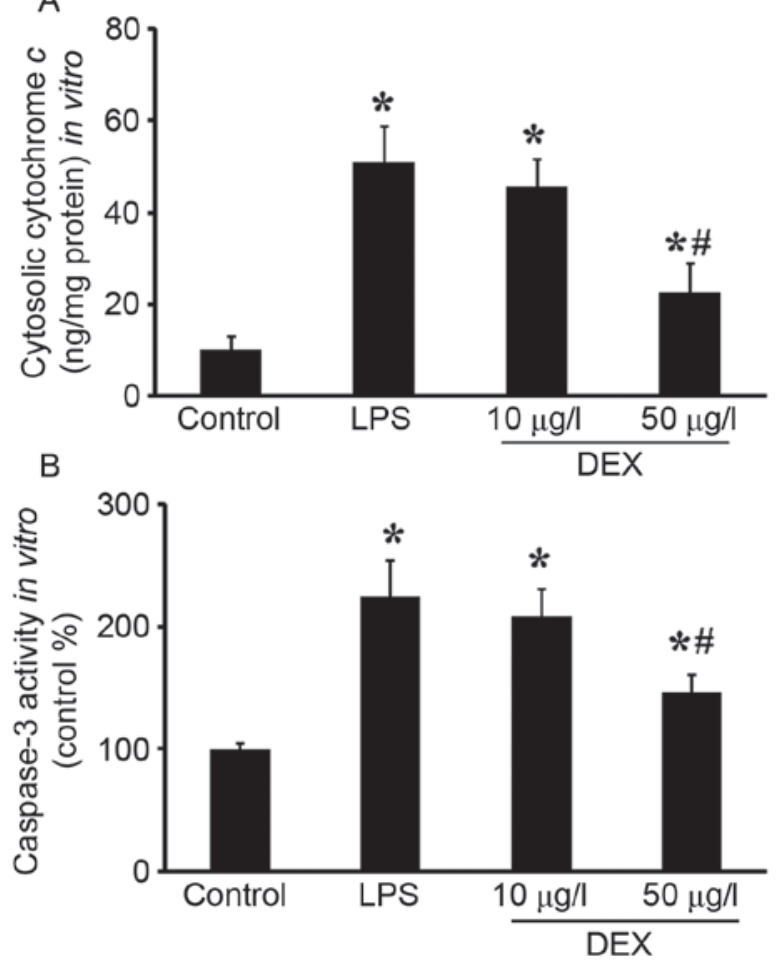

Figure 4. DEX improves LPS-induced cytochrome $c$ release and caspase-3 activation in vitro. (A) The LPS-induced increase in cytosolic cytochrome $c$ was attenuated by DEX treatment. (B) LPS-induced caspase-3 activation was attenuated by DEX treatment. The data are presented as the mean \pm standard deviation ( $\mathrm{n}=6$ /group; ${ }^{*} \mathrm{P}<0.05$ compared with the control group; ${ }^{\#} \mathrm{P}<0.05$ compared with the LPS group). DEX, dexmedetomidine; LPS, lipopolysaccharide.

compared with the LPS group for $50 \mu \mathrm{g} / \mathrm{kg}$ DEX group; P>0.05 compared with the LPS group for $10 \mu \mathrm{g} / \mathrm{kg}$ DEX group; Fig. $5 \mathrm{~A}$ and $\mathrm{C}$ ). Expression of the pro-apoptotic protein Bax was upregulated by LPS induction and inhibited by DEX pretreatment $(\mathrm{P}<0.05$ compared with the LPS group for $50 \mu \mathrm{g} / \mathrm{kg} \mathrm{DEX}$ group; P>0.05 compared with the LPS group for $10 \mu \mathrm{g} / \mathrm{kg}$ DEX group; Fig. 5A and D). These results indicated that intratracheal administration LPS induced lung cell apoptosis, which can be significantly alleviated by treatment with DEX.

DEX alleviates LPS-induced oxidation. Oxidative stress is one of the most important mediators of mitochondrial dysfunction and apoptotic signaling. In vitro, a fluorescent probe, DCFH-DA, was used as a specific marker for quantitative mitochondrial ROS accumulation. LPS-induced mitochondrial ROS production was evidenced by increased intensity of DCF fluorescence in LPS-challenged rats compared with the control animals. This increase was attenuated by pretreatment with $\mathrm{DEX}(50 \mu \mathrm{g} / \mathrm{l})(\mathrm{P}<0.05$ compared with the LPS group for $50 \mu \mathrm{g} / 1 \mathrm{DEX}$ group; $\mathrm{P}>0.05$ compared with the LPS group for $10 \mu \mathrm{g} / 1$ DEX group; Fig. 6A). To evaluate the oxidative stress in vivo, LPO levels were determined in rat serum. The LPO levels significantly increased in the LPS-challenged rats, which was subsequently reduced by pretreatment with DEX $(50 \mu \mathrm{g} / \mathrm{kg})(\mathrm{P}<0.05$ compared with the LPS group for $50 \mu \mathrm{g} / \mathrm{kg}$ DEX group; $\mathrm{P}>0.05$ compared with the LPS group for $10 \mu \mathrm{g} / \mathrm{kg}$ DEX group; Fig. 6B).

\section{Discussion}

Gram-negative organisms account for $\sim 50 \%$ of infections predisposing to ALI, often in the setting of pneumonia or sepsis (27). LPS endotoxin is the critical mediator of the organ dysfunction and mortality associated with severe Gram-negative infections $(29,30)$. It is well-established that intratracheal administration of LPS can induce a model of ALI (4,27). In the present study, to investigate whether DEX attenuates sepsis-induced ALI, ALI rat model was established through intratracheal injection of LPS (5 mg/kg), according to a previous report (4). The present study observed significant lung injuries and dysfunction following LPS administration, evidenced by deterioration of histopathology, increased vascular permeability, W/D weight ratio of the lung and decreased $\mathrm{P}_{\mathrm{a}} \mathrm{O}_{2} / \mathrm{FIO}_{2}$, which is consistent with other studies $(2,4)$. In previous studies, the protective effect of DEX against ALI has been demonstrated in several models $(16,17,19)$. Following pretreatment of DEX $(50 \mu \mathrm{g} / \mathrm{kg})$, LPS-induced ALI was attenuated, which was reflected by improved histopathological changes, vascular permeability, lung water content and $\mathrm{P}_{\mathrm{a}} \mathrm{O}_{2} / \mathrm{FIO}_{2}$.

DEX has been reported to exert protective effect against ischemia/reperfusion injury in isolated rat hearts and may be associated with inhibiting the opening of MPTP (24). In addition, DEX has been demonstrated to induce human neutrophil apoptosis, which is associated with the caspase cascade and loss of MMP (26). To explore the mechanism behind the attenuation of ALI performed by DEX, the mitochondrial function and mitochondrial-dependent apoptosis were investigated in the present study. A reduction in MMP was demonstrated through JC-1 staining. LPS-induced MMP dissipation was reflected by the fluorescence changes in vivo and in vitro, which was subsequently improved by DEX treatment. MMP is dissipated in response to the arrest of the function of the respiratory complexes (I-V), which contributes to an inhibition of ATP biosynthesis. In vitro, DEX inhibited LPS-induced cellular ATP decrease.

Mitochondrial-dependent apoptosis is activated through the mitochondrial release of cytochrome $c$, AIF and Smac (28). Cytochrome $c$, once in the cytosol, acts to release the apoptosome assembly from the apoptotic protease-activating factor 1, ATP and procaspase-9, leading to cellular morphological and functional alterations via the activation of caspase 3 and caspase 7 (13). To further elucidate the present hypothesis of mitochondrial mediated apoptosis, cytosolic cytochrome $c$ levels were estimated with a cytochrome $c$ ELISA kit. It was demonstrated that DEX improved LPS-induced mitochondrial release of cytochrome $c$ into the cytoplasm. Additionally, the present results demonstrated that DEX prevents LPS-induced caspase 3 activation in vivo and in vitro.

Mitochondrial regulation of apoptosis is also regulated by members of the Bcl-2 family of proteins $(31,32)$. The Bcl family consists of both antiapoptotic (Bcl-2, Bcl-xL) and proapoptotic (BAK, Bax) factors. The pro-apoptotic members of this family, including Bax, trigger the release of mitochondrial apoptogenic factors into the cytoplasm by acting on the MPTP, thereby leading to caspases activation. While the antiapoptotic members serve a contrasting role to prevent 
A
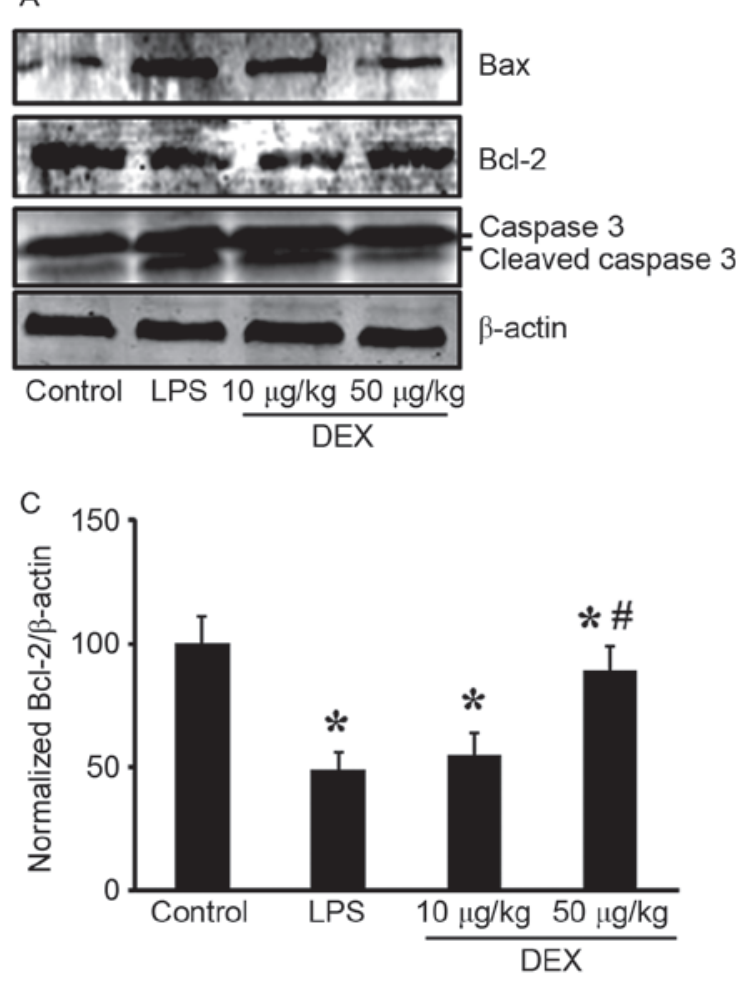

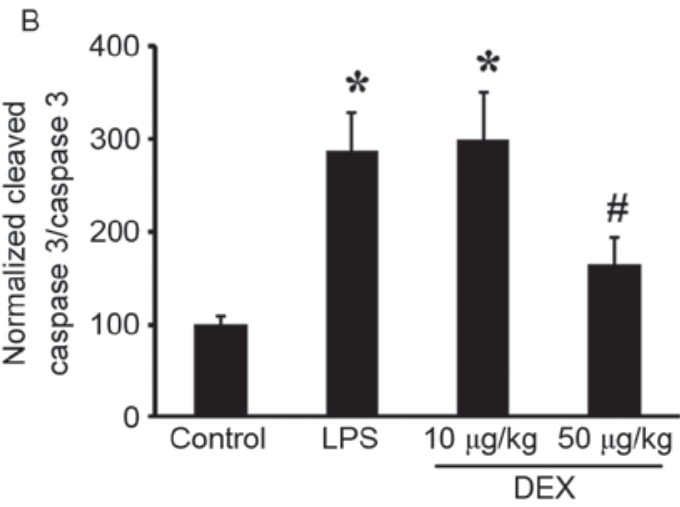

D

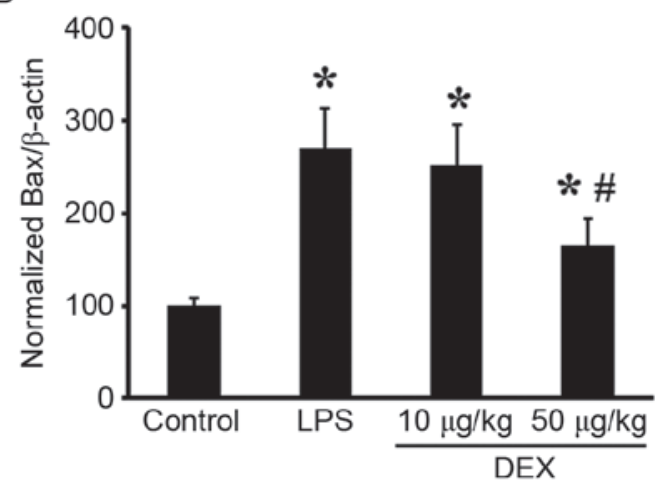

Figure 5. DEX improves LPS-induced downregulation of Bcl-2, and the upregulation of Bax and cleaved caspase 3 in vivo. (A) The expression of Bax, Bcl-2 and cleaved caspase 3 in the lung of control, LPS and DEX (10 and $50 \mu \mathrm{g} / \mathrm{kg}$ ) groups were detected by western blotting. Densitometry was used to quantify the protein expression levels of (B) Bax, (C) cleaved caspase 3 and (D) Bcl-2. B-actin and total caspase 3 were used for normalization. The data are presented as the mean \pm standard deviation ( $\mathrm{n}=6 /$ group; ${ }^{*} \mathrm{P}<0.05$ compared with the control group; ${ }^{*} \mathrm{P}<0.05$ compared with the LPS group). DEX, dexmedetomidine; LPS, lipopolysaccharide; Bcl-2, B-cell lymphoma 2; Bax, Bcl-2-associated X protein.

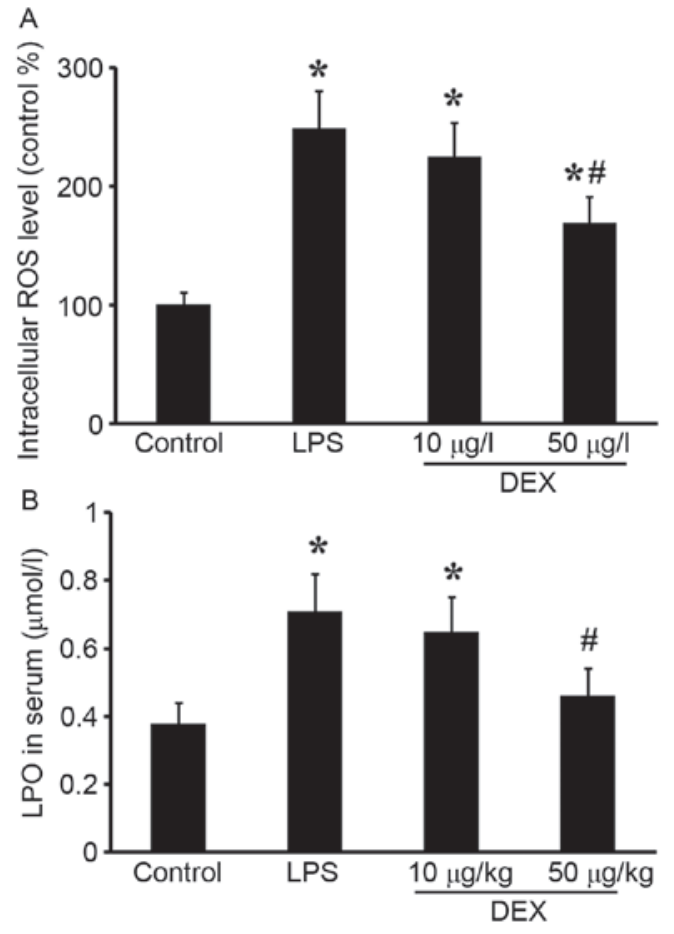

Figure 6. DEX improves LPS-induced oxidative stress. (A) The LPS-induced increase in cellular ROS levels was attenuated by DEX treatment in vitro. (B) The LPS-induced increase in serum LPO levels was improved by DEX treatment. The data are presented as the mean \pm standard deviation ( $\mathrm{n}=6 /$ group; ${ }^{*} \mathrm{P}<0.05$ compared with the control group; ${ }^{*} \mathrm{P}<0.05$ compared with the LPS group). DEX, dexmedetomidine; LPS, lipopolysaccharide; ROS, reactive oxygen species; LPO, lipid peroxides. apoptosis. In the present study, the expression levels of Bax and $\mathrm{Bcl}-2$ were detected by western blotting in the rat samples. The results suggested that LPS-induced upregulation of Bax and downregulation of Bcl-2, which were improved by DEX treatment.

Oxidative stress is one of the most important mediators of mitochondrial dysfunction and apoptotic signaling $(33,34)$. mPTP are major targets of ROS, from which Smac and cytochrome $c$ are released into the cytoplasm after mPTP opening $(7,35)$. The protective effect of DEX has been demonstrated in other diseases. In the present study, DEX inhibits LPS-induced oxidative stress, as evidenced by decreased cellular ROS and serum LPO levels following LPS stimulation. It is quite possible that mitochondrial oxidative stress serves an important role in the release of cytochrome $c$ into the cytoplasm. DEX, by its antioxidant activity, may have prevented this effect.

In conclusion, the present study has provided evidence to suggest that DEX exerted significant attenuation of LPS-induced ALI in a rat model. It was also revealed that the potential mechanism of this action is through amelioration of oxidative stress, mitochondrial dysfunction and mitochondrial-dependent apoptosis.

\section{Acknowledgements}

The present study was supported by the National Natural Science Foundation of China (no. 81500066). 


\section{References}

1. Aschner Y,Zemans RL, Yamashita CM and Downey GP: Matrix metalloproteinases and protein tyrosine kinases: Potential novel targets in acute lung injury and ARDS. CHEST 146: 1081-1091, 2014.

2. Xu Y, Ito T, Fushimi S, Takahashi S, Itakura J, Kimura R, Sato M, Mino M, Yoshimura A and Matsukawa A: Spred-2 deficiency exacerbates lipopolysaccharide-induced acute lung inflammation in mice. PLOS One 9: e108914, 2014.

3. Ito Y, Correll K, Schiel JA, Finigan JH, Prekeris R and Mason RJ: Lung fibroblasts accelerate wound closure in human alveolar epithelial cells through hepatocyte growth factor/c-Met signaling. Am J Physiol Lung Cell Mol Physiol 307: L94-L105, 2014.

4. Gonzales JN, Gorshkov B, Varn MN, Zemskova MA, Zemskov EA, Sridhar S, Lucas R and Verin AD: Protective effect of adenosine receptors against lipopolysaccharide-induced acute lung injury. Am J Physiol Lung Cell Mol Physiol 306: L497-L507, 2014.

5. Martínez-González I, Roca O, Masclans JR, Moreno R, Salcedo MT, Baekelandt V, Cruz MJ, Rello J and Aran JM: Human mesenchymal stem cells overexpressing the IL-33 antagonist soluble IL-1 receptor-like-1 attenuate endotoxin-induced acute lung injury. Am J Respir Cell Mol Biol 49: 552-562, 2013.

6. Wang W, Xie Q, Zhou X, Yao J, Zhu X, Huang P, Zhang L, Wei J, Xie H, Zhou L and Zheng S: Mitofusin-2 triggers mitochondria $\mathrm{Ca} 2+$ influx from the endoplasmic reticulum to induce apoptosis in hepatocellular carcinoma cells. Cancer Lett 358: $47-58,2015$.

7. Sinha K, Das J, Pal PB and Sil PC: Oxidative stress: The mitochondria-dependent and mitochondria-independent pathways of apoptosis. Arch Toxicol 87: 1157-1180, 2013.

8. Liu G, Zhang J, Chen H, Wang C, Qiu Y, Liu Y, Wan J and Guo H: Effects and mechanisms of alveolar type II epithelial cell apoptosis in severe pancreatitis-induced acute lung injury. Exp Ther Med 7: 565-572, 2014

9. Bhandari V, Choo-Wing R, Lee CG, Zhu Z, Nedrelow JH, Chupp GL, Zhang X, Matthay MA, Ware LB, Homer RJ, et al Hyperoxia causes angiopoietin 2-mediated acute lung injury and necrotic cell death. Nat Med 12: 1286-1293, 2006.

10. Kujoth GC, Hiona A, Pugh TD, Someya S, Panzer K, Wohlgemuth SE, Hofer T, Seo AY, Sullivan R, Jobling WA, et al: Mitochondrial DNA mutations, oxidative stress, and apoptosis in mammalian aging. Science 309: 481-484, 2005.

11. Green DR and Kroemer G: The pathophysiology of mitochondria cell death. Science 305: 626-629, 2004

12. Khalil WK, Assaf N, ElShebiney SA and Salem NA Neuroprotective effects of bee venom acupuncture therapy against rotenone-induced oxidative stress and apoptosis. Neurochem int 80: 79-86, 2015.

13. Lakhani SA, Masud A, Kuida K, Porter GA Jr, Booth CJ, Mehal WZ, Inayat I and Flavell RA: Caspases 3 and 7: Key mediators of mitochondrial events of apoptosis. Science 311 847-851, 2006

14. Chen CH, Chen SJ, Su CC, Yen CC, Tseng TJ, Jinn TR, Tang FC, Chen KL, Su YC, Lee kI, et al: Chloroacetic acid induced neuronal cells death through oxidative stress-mediated p38-MAPK activation pathway regulated mitochondria-dependent apoptotic signals. Toxicology 303: 72-82, 2013.

15. Singh BK, Tripathi M, Chaudhari BP, Pandey PK and Kakkar P: Natural terpenes prevent mitochondrial dysfunction, oxidative stress and release of apoptotic proteins during nimesulide-hepatotoxicity in rats. Plos One 7: e34200, 2012.

16. Chen C, Zhang Z, Chen K, Zhang F, Peng M and Wang Y: Dexmedetomidine regulates inflammatory molecules contributing to ventilator-induced lung injury in dogs. J Surg Res 187: 211-218, 2014.

17. Gu J, Chen J, Xia P, Tao G, Zhao H and Ma D: Dexmedetomidine attenuates remote lung injury induced by renal ischemia-reperfusion in mice. Acta Anaesthesiol Scand 55: 1272-1278, 2011.
18. Cavalcanti V, Santos CL, Samary CS, Araújo MN, Heil LB, Morales MM, Silva PL, Pelosi P, Fernandes FC, Villela N and Rocco PR: Effects of short-term propofol and dexmedetomidine on pulmonary morphofunction and biological markers in experimental mild acute lung injury. Respir Physiol Neurobiol 203: 45-50, 2014

19. Sen V, Guzel A, Sen HS, Ece A, Uluca U, Söker S, Doğan E, Kaplan İ and Deveci E: Preventive effects of dexmedetomidine on the liver in a rat model of acid-induced acute lung injury. Biomed Res Int 2014: 621827, 2014.

20. Hwang L, Choi IY, Kim SE, Ko IG, Shin MS, Kim CJ, Kim SH, Jin JJ, Chung JY and Yi JW: Dexmedetomidine ameliorates intracerebral hemorrhage-induced memory impairment by inhibiting apoptosis and enhancing brain-derived neurotrophic factor expression in the rat hippocampus. Int J Mol Med 31: 1047-1056, 2013

21. Zhang XY, Liu ZM, Wen SH, Li YS, Li Y, Yao X, Huang WQ and Liu KX: Dexmedetomidine administration before, but not after, ischemia attenuates intestinal injury induced by intestinal ischemia-reperfusion in rats. Anesthesiology 116: 1035-1046, 2012.

22. Xiang H, Hu B, Li Z and Li J: Dexmedetomidine controls systemic cytokine levels through the cholinergic anti-inflammatory pathway. Inflammation 37: 1763-1770, 2014.

23. Zhang X, Wang J, Qian W, Zhao J, Sun L, Qian Y and Xiao H: Dexmedetomidine inhibits tumor necrosis factor-alpha and interleukin 6 in lipopolysaccharide-stimulated astrocytes by suppression of c-Jun N-terminal kinases. Inflammation 37: 942-949, 2014.

24. Jiang C, Xia M, Wang M and Chen S: Dexmedetomidine preconditioning protects isolated rat hearts against ischemia/reperfusion injuries and its mechanism. Zhejiang Da Xue Xue Bao Yi Xue Ban 42: 326-330, 2013 (In Chinese).

25. Chiu KM, Lin TY, Lu CW and Wang SJ: Inhibitory effect of glutamate release from rat cerebrocortical nerve terminals by $\alpha 2$ adrenoceptor agonist dexmedetomidine. Eur J Pharmacol 670: 137-147, 2011

26. Kishikawa H, Kobayashi K, Takemori K, Okabe T, Ito K and Sakamoto A: The effects of dexmedetomidine on human neutrophil apoptosis. Biomed Res 29: 189-194, 2008.

27. Xie K, Yu Y, Huang Y, Zheng L, Li J, Chen H, Han H, Hou L, Gong $\mathrm{G}$ and Wang $\mathrm{G}$ : Molecular hydrogen ameliorates lipopolysaccharide-induced acute lung injury in mice through reducing inflammation and apoptosis. Shock 37: 548-555, 2012.

28. Childs EW, Tharakan B, Hunter FA, Tinsley JH and Cao X Apoptotic signaling induces hyperpermeability following hemorrhagic shock. Am J Physiol Heart Circ Physiol 292: H3179-H3189, 2007.

29. Kumpf O, Giamarellos-Bourboulis EJ, Koch A, Hamann L, Mouktaroudi M, Oh DY, Latz E, Lorenz E, Schwartz DA, Ferwerda B, et al: Influence of genetic variations in TLR4 and TIRAP/Mal on the course of sepsis and pneumonia and cytokine release: An observational study in three cohorts. Crit Care 14: R103, 2010

30. Saluk-Juszczak J and Wachowicz B: The proinflammatory activity of lipopolysaccharide. Postepy Biochem 51: 280-287, 2005 (In Polish)

31. Renault TT, Floros KV, Elkholi R, Corrigan KA, Kushnareva Y, Wieder SY, Lindtner C, Serasinghe MN, Asciolla JJ, Buettner C, et al: Mitochondrial shape governs BAX-induced membrane permeabilization and apoptosis. Mol Cell 57: 69-82, 2015.

32. Llambi F and Green DR: Apoptosis and oncogenesis: Give and take in the BCL-2 family. Curr Opin Genet Dev 21: 12-20, 2011.

33. Liu Y,Zeng X, Hui Y, Zhu C, Wu J, Taylor DH, Ji J, Fan W, Huang Z and $\mathrm{Hu} \mathrm{J}$ : Activation of $\alpha 7$ nicotinic acetylcholine receptors protects astrocytes against oxidative stress-induced apoptosis: Implications for Parkinson's disease. Neuropharmacology 91 87-96, 2015.

34. Matés JM, Segura JA, Alonso FJ and Márquez J: Oxidative stress in apoptosis and cancer: An update. Arch Toxicol 86: 1649-1665, 2012

35. Wang X, Song R, Chen Y, Zhao M and Zhao K: Polydatin-a new mitochondria protector for acute severe hemorrhagic shock treatment. Expert Opin Inv Drug 22: 169-179, 2013. 